\title{
NON-DESTRUCTIVE MONITORING STRATEGIES OF HISTORICAL CONSTRUCTIONS AND TANGIBLE CULTURALE HERITAGE BASED ON GROUND-BASED SAR INTERFEROMETRY
}

\begin{abstract}
Tangible cultural heritage, historical buildings and bridges have an important cultural significance and economic value within the tourism industry and the identity of local communities. The preservation and the assessment of their structural health are important issues which call for multidisciplinary teams and non-invasive monitoring techniques due the uniqueness and historical values of these man-made structures. Numerical models used to study the structural behavior of these historical buildings and bridges under different adverse conditions (e.g. intense traffic flow, natural hazard events, chemical pollution or simply aging) can benefit from accurate measurements of mechanical properties such as displacements and vibration frequencies, both bringing information about the static and dynamical behavior of such historical constructions. This work presents some results of structural monitoring of man-made structures by Ground-based Synthetic Aperture Radar (GBSAR) interferometry techniques. A ku-band GBSAR interferometer is used to derive displacement maps of the monitored target, with a sub-millimeter precisions. Furthermore, GBSAR interferometry is used to measure vibration frequencies of vertical and horizontal structures, such bell towers, towers, bridges and historical walls. The main advantage of this technique is its capability to operate in any weather and sun-illumination condition, in a truly Non-Destructive Monitoring (NDM) approach, i.e. without installing any reflector on the observed target.
\end{abstract}

KEYWORDS: Synthetic Aperture Radar (SAR), Ground-based SAR (GBSAR), SAR interferometry, Non-Destructive Monitoring (NDM), Structural Health Monitoring (SHM), metrology, displacement, vibration frequency, monuments, cultural heritage.

\section{INTRODUCTION}

The preservation of historical constructions and heritage sites is a major societal and scientific challenge of many countries. This becomes particular important in countries prones to natural hazards, against natural hazards, such as extreme climate events, floodings and earthquakes [Fanti et al., 2013]. There is a need for Non-Destructive Monitoring (NDM) methodologies that can provide quantitative information about the structural health of historical constructions and heritage sites, and that are cost-effective in order to set plans of regular periodical measurement campaigns or to easily carry out emergency check-ups after serious natural hazards events [Masini et al., 2010; Chen et al., 2018].

The preservation of historical constructions and heritage sites is highly stimulating intersectorial and multidisciplinary research fields, needing both numerical modeling and analysis of structures and measurements of different physical and chemical properties of both structures and construction materials.

\footnotetext{
${ }^{1}$ Consiglio Nazionale delle Ricerche, Istituto per le Applicazioni del Calcolo, Via Amendola, 122/O, 75100, Bari, Italy, e-mail: g.nico@ba.iac.cnr.it

${ }^{2}$ Saint Petersburg State University, $10^{\text {th }}$ line V.O., 33, 199178, Saint Petersburg, Russia, e-mail: giovanni.nico@ spbu.ru

3 DIAN srl, Via G.B Pirelli sn, Z.I. La Martella, 75100 Matera, Italy, e-mail: o.masci@dianalysis.eu

${ }^{4}$ Saint Petersburg State University, $10^{\text {th }}$ line V.O., 33, 199178, Saint Petersburg, Russia, e-mail: panidi@ya.ru, e.panidi@spbu.ru
} 
This work is aimed at presenting some results of ongoing research activities concerning the measurement of deformations and vibration frequencies by Ground-Based Synthetic Aperture Radar (GBSAR) interferometers for structural assessment of monumental buildings. Results presented in the work were acquired at two test beds affected by various forms of material and structural degradation. These are: the Modena's Ghirlandina belltower, and the Orvieto's Cathedral façade, both in Italy.

GBSAR interferometry makes possible to obtain dense and precise (sub-millimetre) deformation measurements and vibration frequency spectra of each structural element of the historical site at relatively low cost [Pieraccini et al., 2015; Di Pasquale et al., 2018]. For these characteristics, this technology can be a powerful tool, complementary to in-situ measurements and structural analysis technologies, for monitoring of historical sites. The identification of anomalies can possibly indicate the activation of local failure mechanisms and be useful for a detailed architectural and damage survey, as well as in light of damage scenarios predicted by finite element simulations. Furthermore, GBSAR measurements, complemented by visual inspections, can guide the definition and installation of a mixed static-dynamic continuous structural health monitoring (SHM) systems.

\section{GROUND-BASED RADAR INTERFEROMETRY: BASIC PRINCIPLES AND APPLICATIONS}

The ground-based radar interferometry technique relies on a radar system that observes the same scene from the same location at different times. The basics concept is that of Real Aperture Radar (RAR) system which is a stepped-frequency continuous-wave (SF-CW) radar that emits a continuous wave with different progressive frequencies within a given frequency band. The corresponding echoes, backscattered by the scene give rise, to the raw data. If the radar system is moved along a rail of finite length, changing its position with a constant step, a SAR system is obtained where the SF-CWs, with increasing frequencies, are transmitted, and the corresponding echoes received, at each position of the rail. In the case of a SAR system, the raw data structure consists of a matrix with the number of columns, given by the acquisition positions along the rail, and rows, given by the transmitted frequencies. The Frequency-Domain Back-Propagation (FDBA) algorithm provides the exact focusing method of GBSAR. It performs a coherent summation, of the different frequency contributions for each radar position, corrected for the phase delay:

$$
S(\rho, \psi)=\frac{1}{M_{R} N_{R}} \sum_{n=1}^{N_{R}} \sum_{m=1}^{M_{R}} d\left(x_{n}, f_{m}\right) \times \exp \left\{i 4 \pi f_{m} \frac{R}{c}\right\}
$$

where $d(x n, f m)$ is the raw data matrix, $x_{n}$ and $f_{m}$ are the acquisition position and transmitted frequency, respectively, $R$ is the distance of each point in the scene with respect to the center of the synthetic aperture, c is the speed of light and $S(\rho, \psi)$ is the focused image given in polar coordinates. If the $\mathrm{TX} / \mathrm{RX}$ antennas are sled along rail, a SAR image can be created with an azimuth resolution $\Delta x$ :

$$
\Delta x=\frac{\lambda R}{2 L}
$$

where $R$ is the range distance and $L$ is the rail length (in this case $L=2 \mathrm{~m}$ ). In interferometric applications the radar system acquires a time series $\left\{S_{i}, i=1, \ldots, N\right\}$ of either coherent 1D radar profiles or 2D SAR images of the scene. Couples of interferometric radar data can be selected taking consecutive acquisitions or obtained at different times. In both cases, for each pair of radar data the interferometric phase is computed as follows:

$$
\Delta \varphi_{1,2}=\operatorname{atan}\left\{\mathrm{S}_{2} \cdot \operatorname{conj}\left(\mathrm{S}_{1}\right)\right\}
$$

where $S_{1}$ and $S_{2}$ are the two coherent complex-values radar data acquired at times $t_{1}$ and $t_{2}$, respectively. The Line-of-Sight (LoS) displacement $D_{1,2}$ of a point $P$ in the scene, occurred in the time interval $\left[t_{1}, t_{2}\right]$ is related to the interferometric phase $\Delta \varphi_{1,2}$ by the relationship: 


$$
D_{1,2}=\frac{\lambda}{4 \pi} \Delta \varphi_{1,2}
$$

where $\lambda$ is the radar wavelength (in this case $\lambda=18 \mathrm{~mm}$ ). The precision of displacement measurements depends on the accuracy of phase measurements and it can be a fraction of millimeter, if artifacts due to phase propagation in atmosphere are identified and corrected. In the case of RAR interferometric applications, radar data acquired with the minimum sampling time, in the order of a fraction of second, are interferometrically processed to accurately track in time the deformation profile and to measure the vibration frequency spectrum and acceleration of the displacements, an useful information when this technology is applied to the study of dams. In contrast, in SAR interferometric applications the acquisition of SAR images requires a few minutes and the interferometric couples can be formed using a temporal baseline larger than the minimum time interval between the acquisition of two successive SAR images. The reason for this choice can related to the particular monitoring, continuous vs repeated campaign, or to the need to optimize the interferometric processing (e.g. increase the interferometric coherence or mitigate atmospheric phase delay effects).

The research, described in this paper, was carried out using the IBIS-S/L sensor, a commercial radar with interferometric capability built by IDS. The system consists of a sensor module, a control PC, a power supply unit and data acquisition software. The IBIS sensor is a stepped frequency continuous wave radar which transmits an electromagnetic signal at a central frequency of $17.2 \mathrm{GHz}$ (Ku band) with a maximum bandwidth of $300 \mathrm{MHz}$, corresponding to a range resolution of $0.75 \mathrm{~m}$. A description of the GBSAR data acquisition strategies, processing algorithms and visualization tool of measurements can be found in [Di Pasquale et al., 2018].

\section{RESULTS}

In this section we present a few results about the monitoring of monuments in Italy, using the GBSAR technique. In the following, we describe the application of GBSAR interferometry to measure displacements and vibration frequencies of belltowers and pinnacles in the façade of churches. The first example refers to the Ghirlandina belltower, the symbol of the city of Modena, Italy. The Ghirlandina belltower, the Cathedral and the nearby Piazza Grande have been a UNESCO World Heritage Site since 1997. The belltower is 89.32 metres tall and is characterized by strong visible deformations due to a complex construction history, the peculiar subsoil conditions and the effects of both natural and man induced subsidence [Castagnetti et al., 2017]. The aim of the measurement campaign was to demonstrate the use of GBSAR for non-destructive measurements of both the natural vibration frequencies of the belltower structure and those induced by the bell ringing. Figure 1 shows a photo of the Ghirlandina belltower and of the GBSAR system.
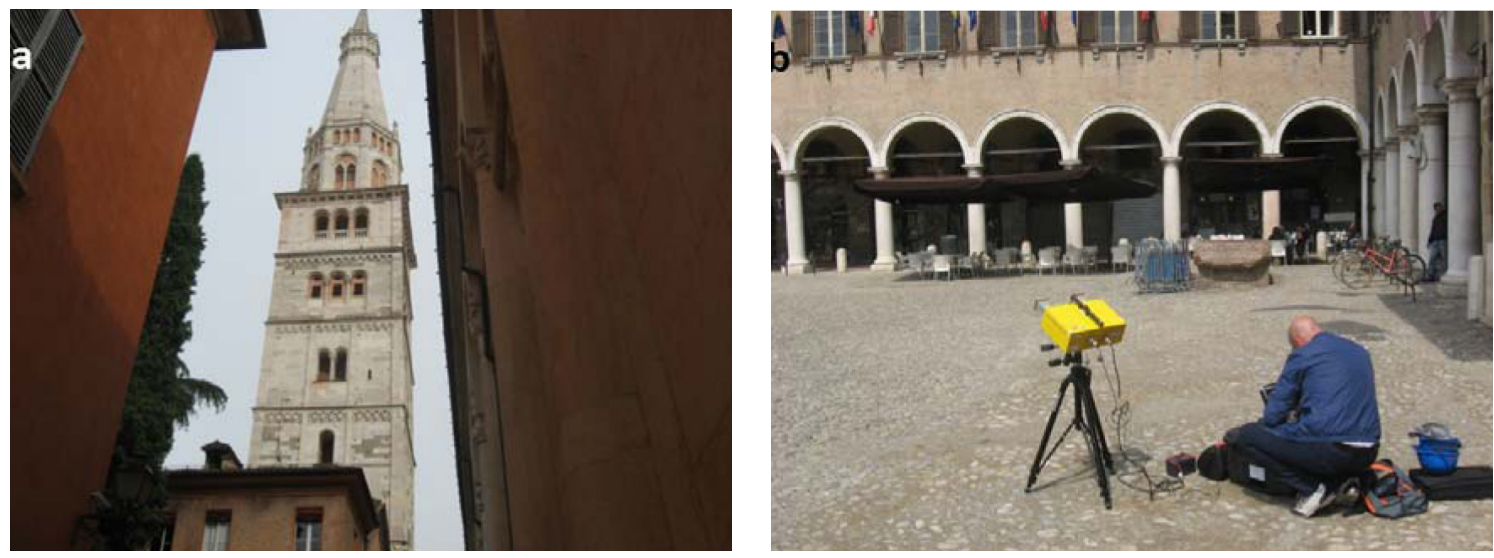

Fig. 1. (a) Photo of the Ghirlandina belltower from one of the GBSAR acquisition stations;

(b) detail of the GBSAR system used in the measurement campaign 
The following Figure 2 and 3 show the results which were observed by two different GBSAR installation sites. In particular, Figure 2a reports the profile of Radar Cross Section (RCS), i.e. the radar response of different targets, observed by the GBSAR. Targets are indentified in terms of their range distance from the GBSAR. For each target, the GBSAR measures the temporal evolution of the LoS displacements. Figure $2 b$ shows the vibration spectrum of all the targets observed by the GBSAR. Examples of LoS displacements and corresponding vibration spectrum of targets of interest located at the top of the belltower are reported in Figures $2 \mathrm{c}, 2 \mathrm{~d}$.
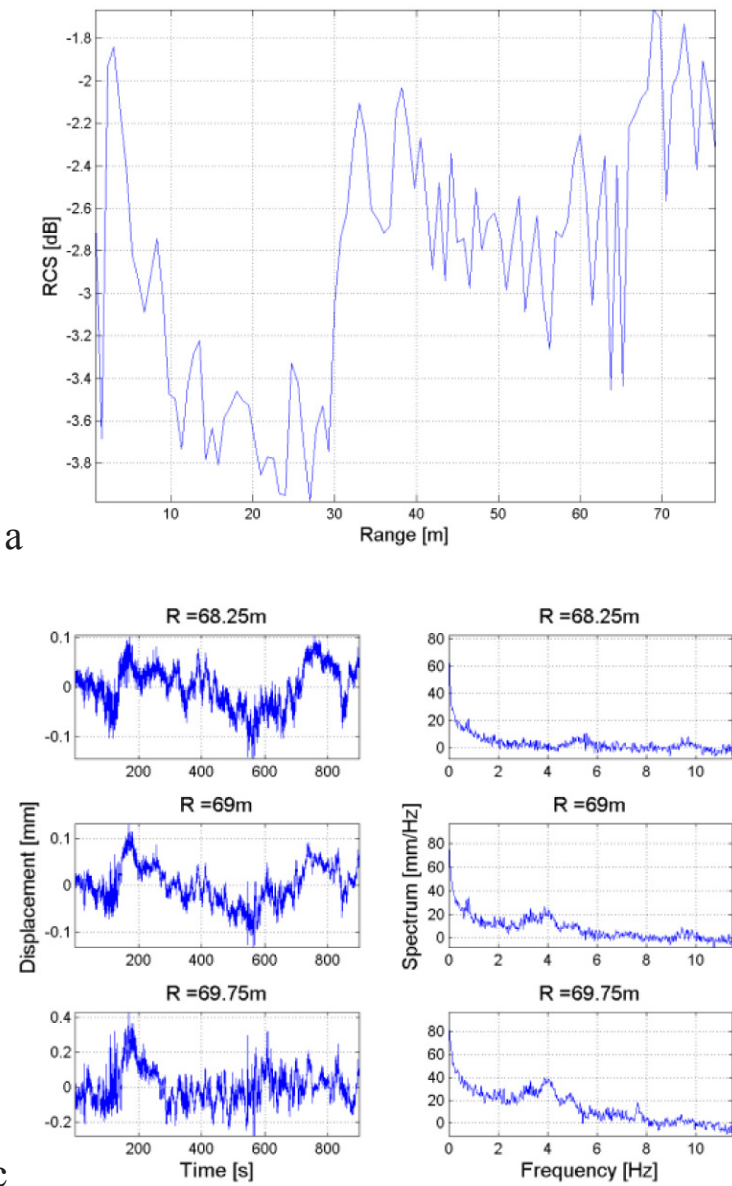

C
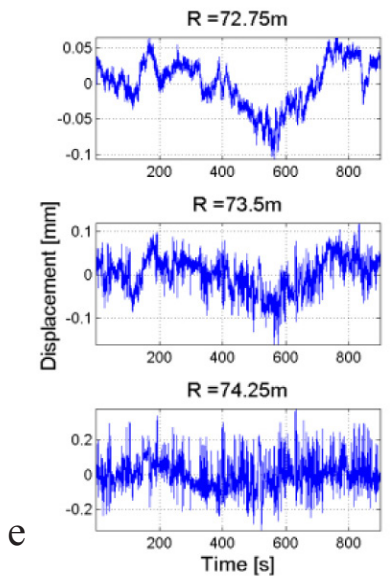
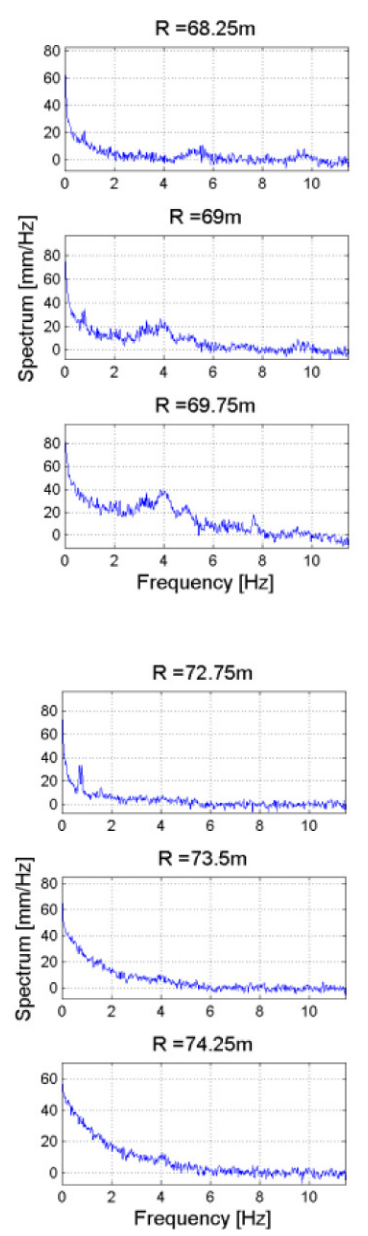

b
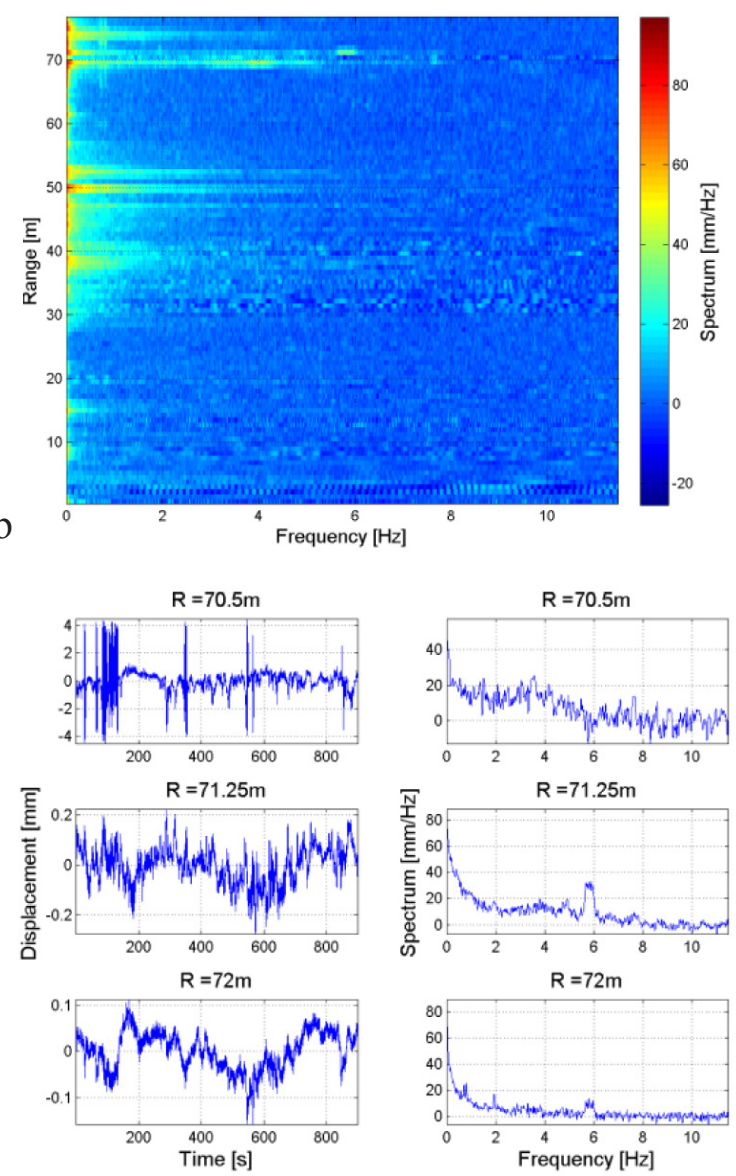

d
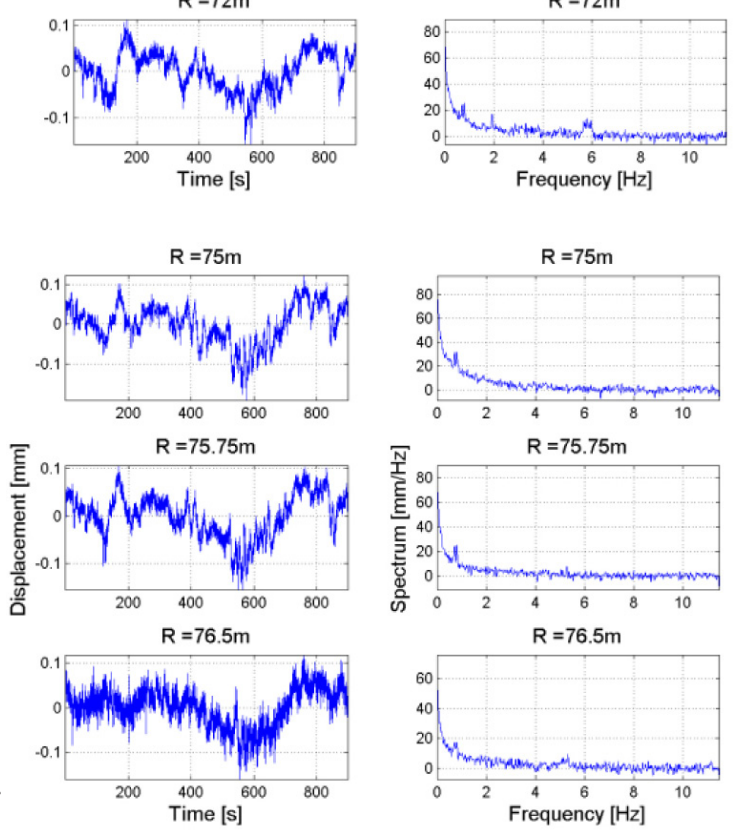

Fig. 2. Ghirlandina belltower, GBSAR site 1:

(a) Radar Cross Section of targets located at range distances up to $80 \mathrm{~m}$; (b) Vibration spectrum of targets; $(\mathrm{c}-\mathrm{f})$ Displacement profiles and the corresponding vibration spectra of selected targets 
a

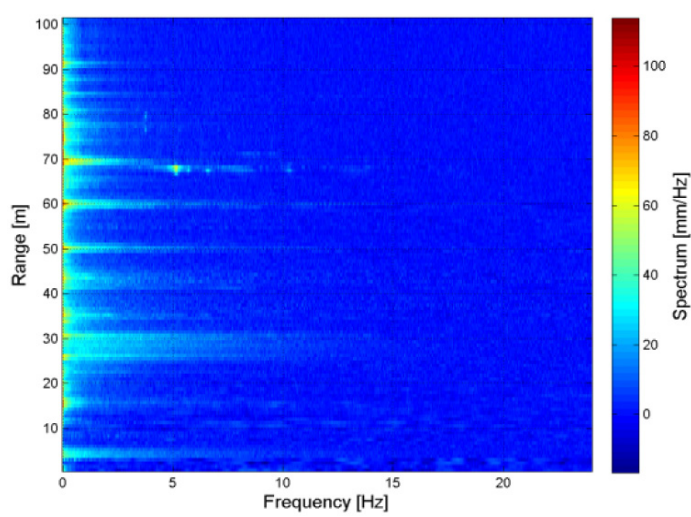

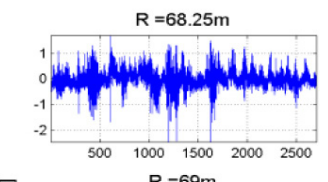
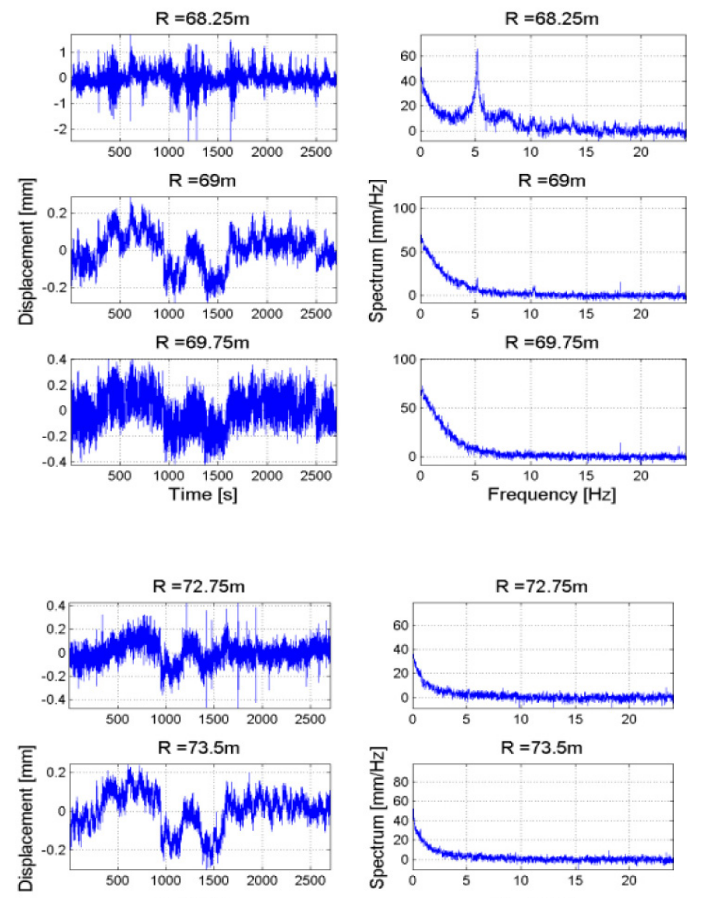
$R=74.25 \mathrm{~m}$
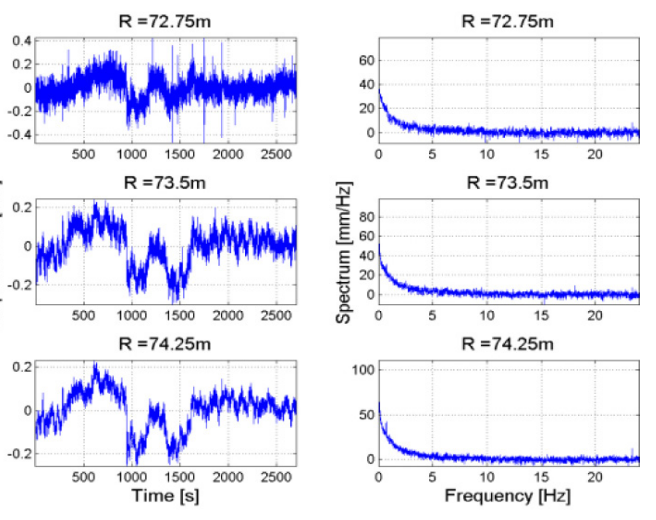

e
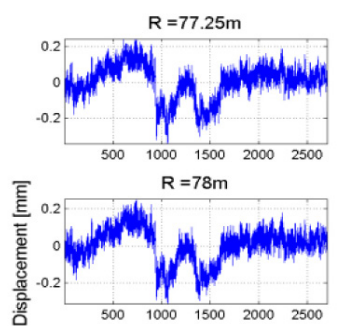

$R=78.75 m$

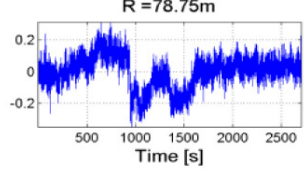

g
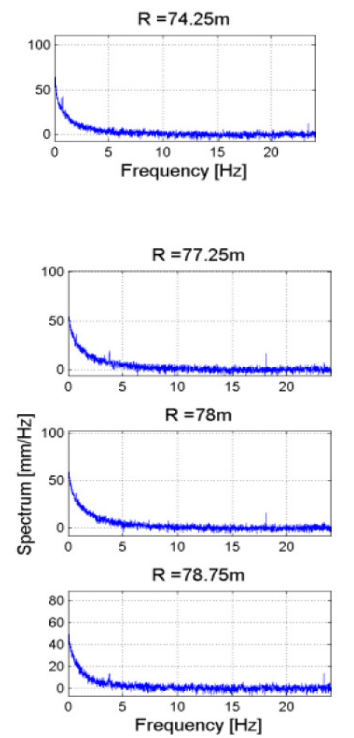

b
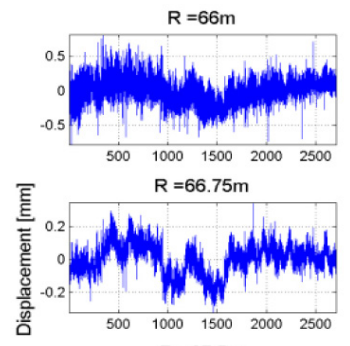

$R=67.5 \mathrm{~m}$
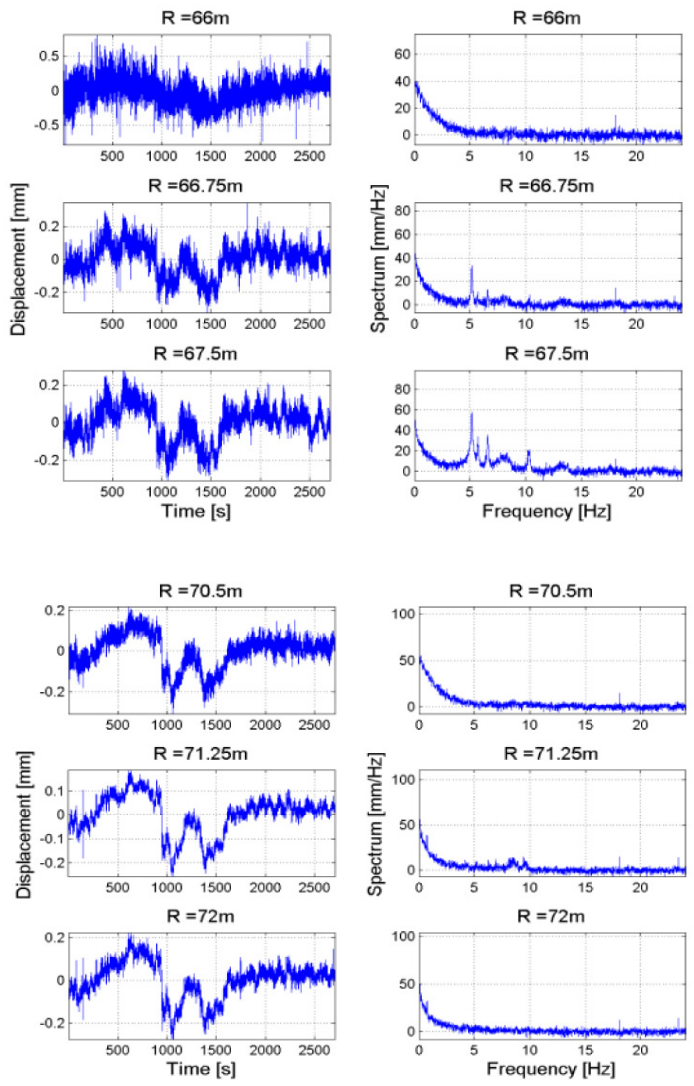

d
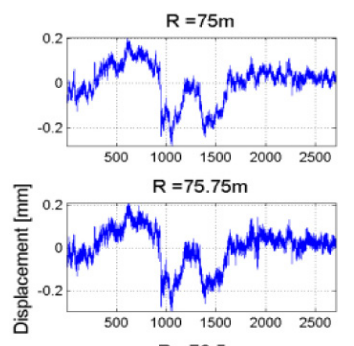

$\mathrm{R}=76.5 \mathrm{~m}$
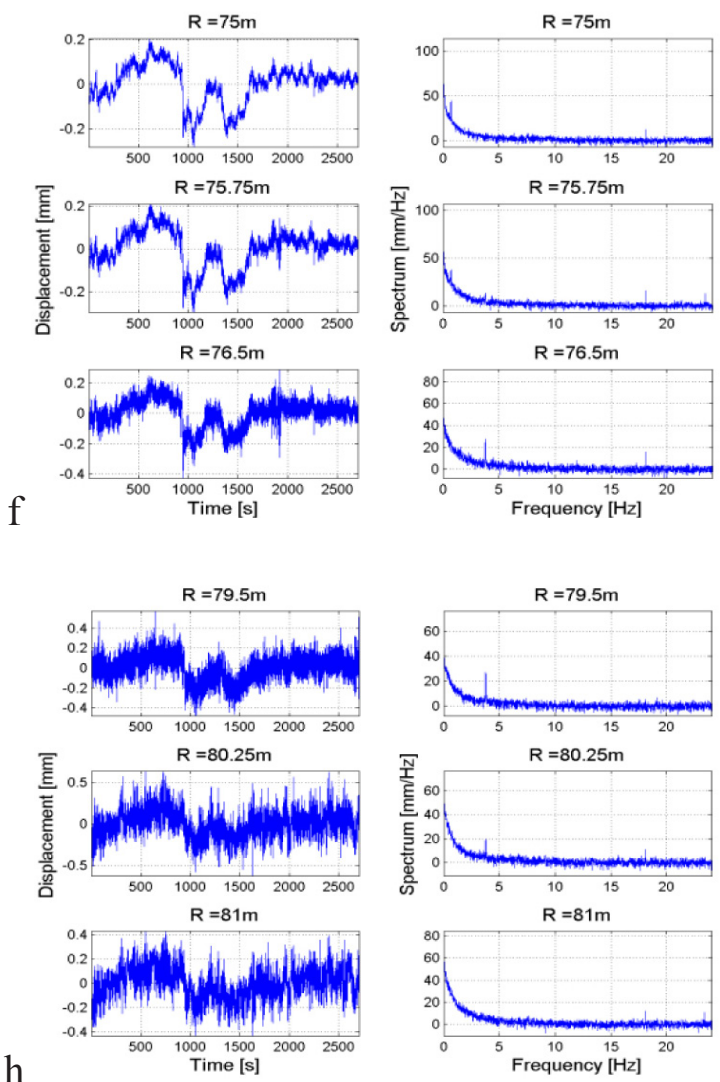

Fig. 3. Ghirlandina belltower, GBSAR site 2:

(a) Radar Cross Section of targets located at range distances up to $80 \mathrm{~m}$; (b) Vibration spectrum of targets; $(\mathrm{c}-\mathrm{f})$ Displacement profiles and the corresponding vibration spectra of selected targets 
Figure 3a reports the vibration spectrum of all the targets observed by the GBSAR at the installation site 2. Examples of LoS displacements and corresponding vibration spectrum of targets of interest located at the top of the belltower are reported in Figures 3b-3h. This dataset was acquired during the ringing of bells and allows to measure the response of the different structural elements of the belltower. In particular, targets located at range distance between $\mathrm{R}=66 \mathrm{~m}$ and $\mathrm{R}=69 \mathrm{~m}$ show vibration frequencies in the range [5 Hz, $10 \mathrm{~Hz}]$, not observed in Figure 2.

The second example refers to the monitoring of the Gothic façade of the Orvieto Cathedral, Italy. The monitored façade and one of the two GBSAR installation sites are shown in Figure 4. The RCS profile and the vibration spectrum of all targets observed by GBSAR at site 1 are reported in Figures 5a and 5b. Examples of LoS displacement and the corresponding vibration spectra are reported in Figure 6.

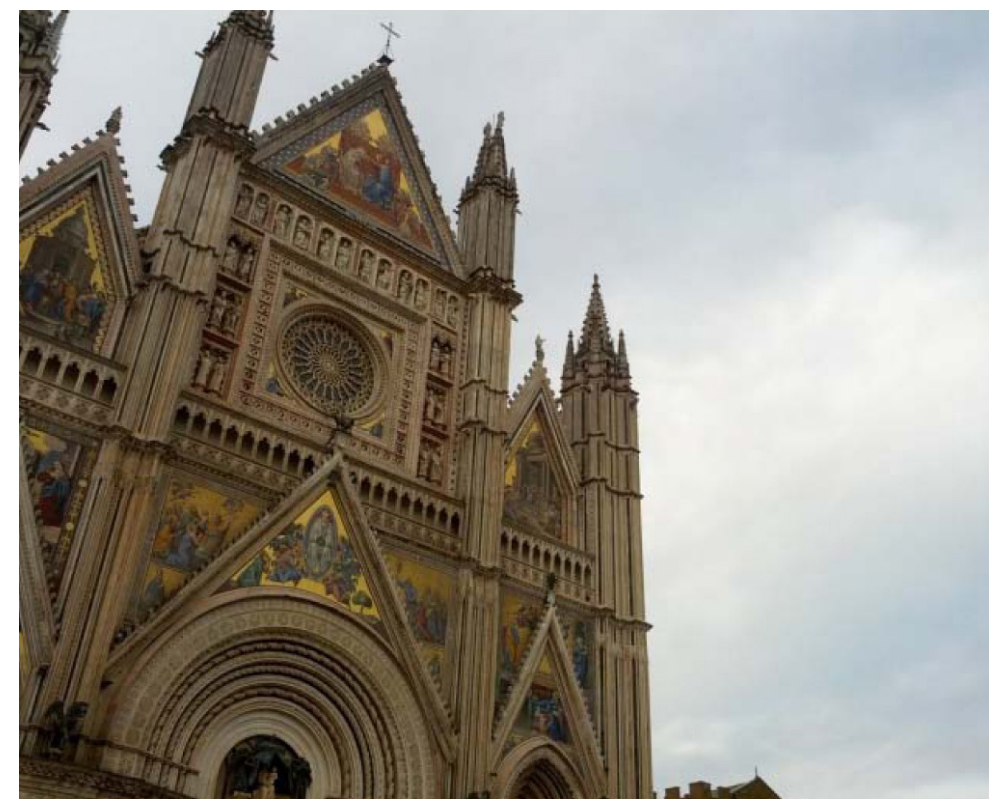

a

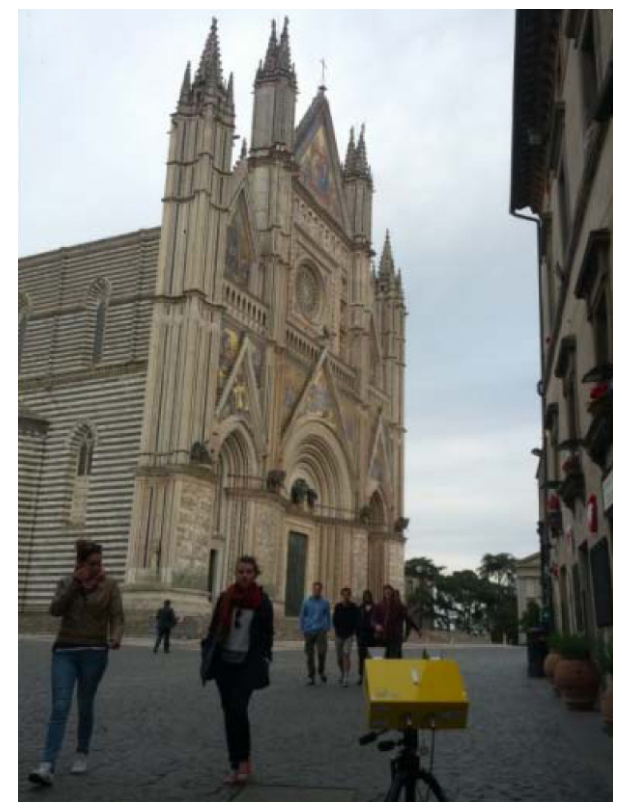

b

Fig. 4. (a) Photo of the Orvieto Cathedral façade; (b) GBSAR installation site 1

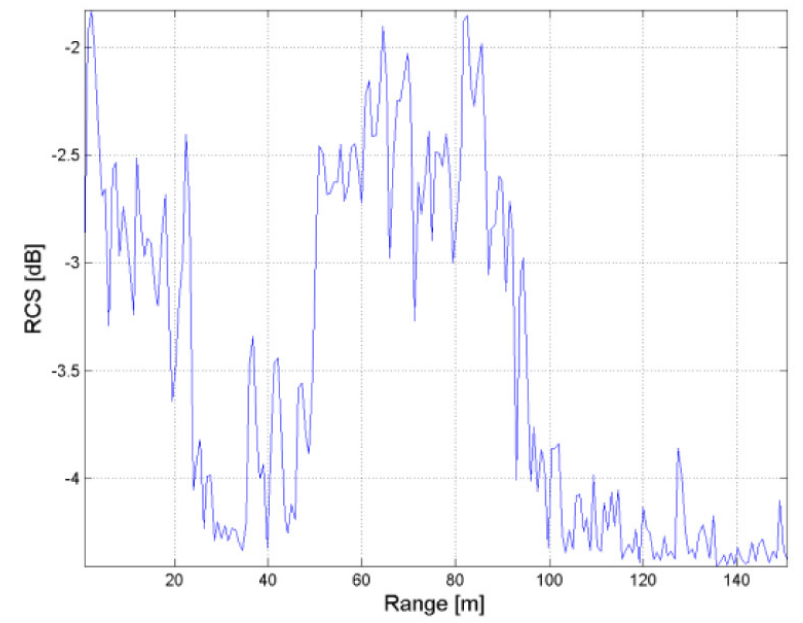

a

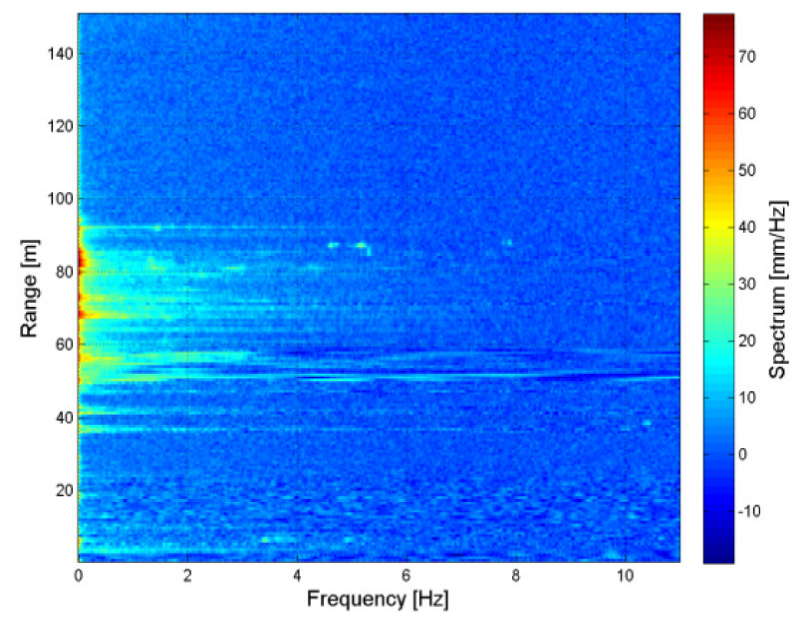

b

Fig. 5. Orvieto Cathedral façade, GBSAR installation site 1:

(a) Radar Cross Section of targets located at range distances up to $150 \mathrm{~m}$; (b) Vibration spectrum of targets 

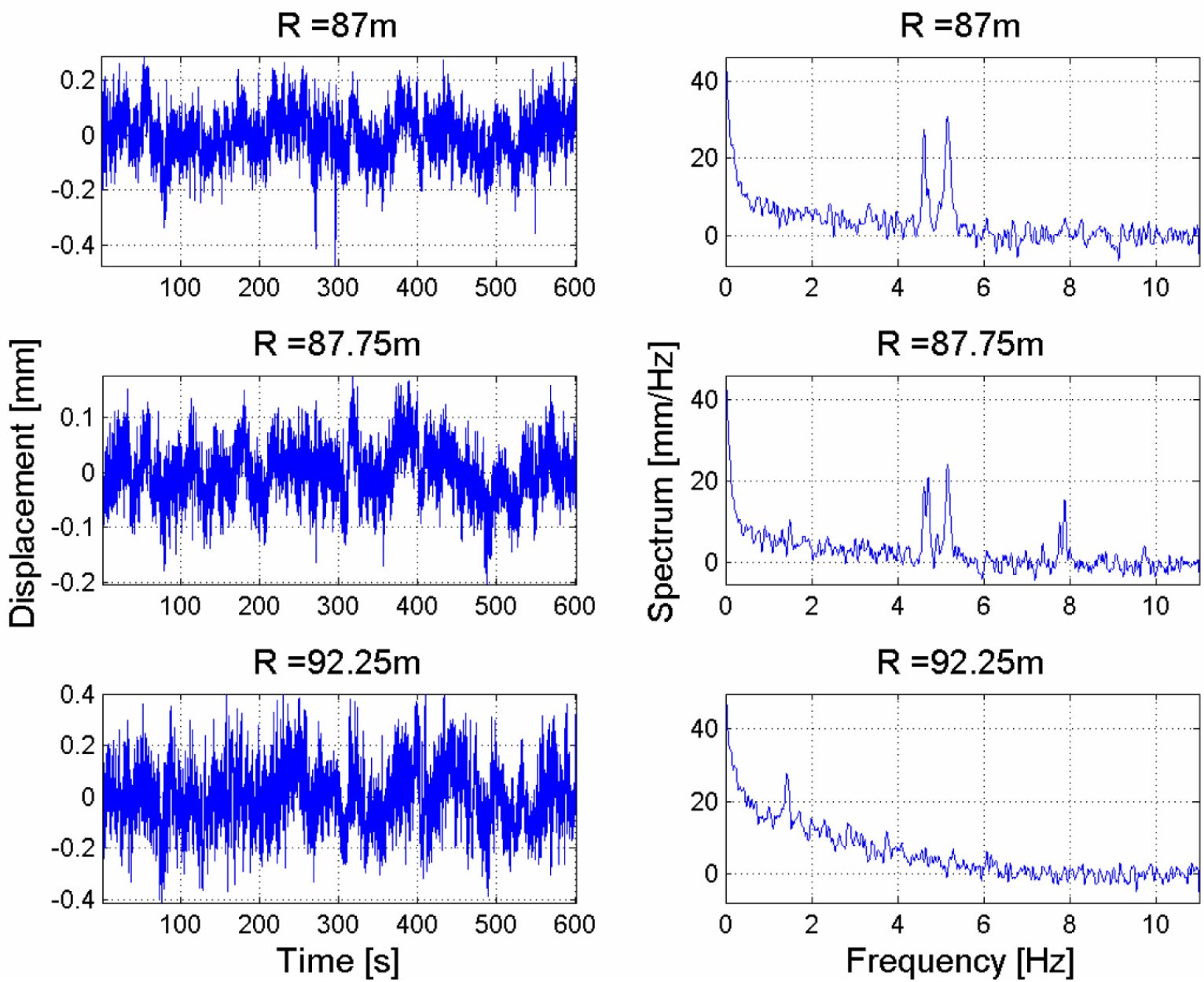

Fig. 6. Orvieto Cathedral façade, GBSAR installation site 1:

displacement profiles and the corresponding vibration spectra of selected targets

The RCS profile and the vibration spectrum of all targets observed by GBSAR at site 2 are reported in Figures 7a and 7b. Examples of LoS displacement and the corresponding vibration spectra are reported in Figure 8.

a

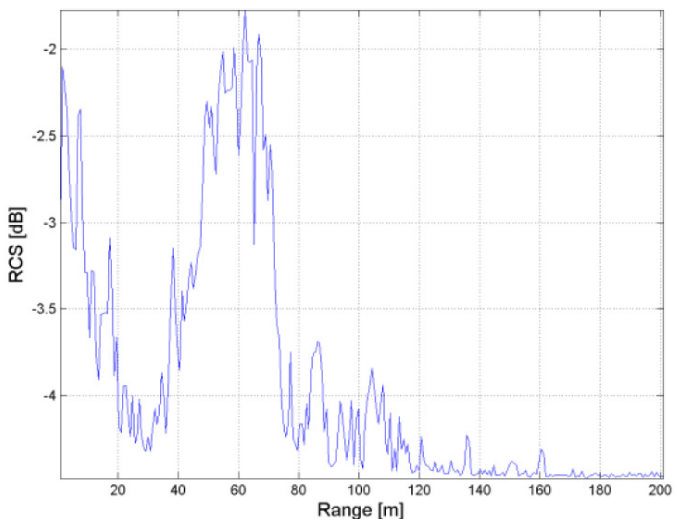

$\mathrm{b}$

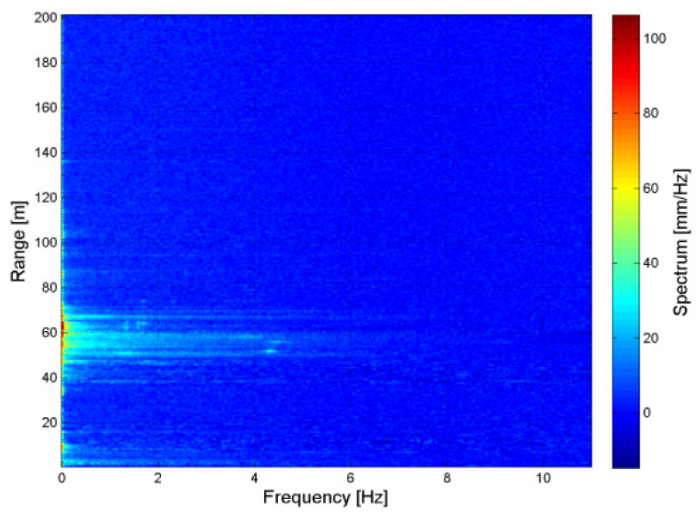

Fig. 7. Orvieto Cathedral façade, GBSAR installation site 2:

(a) Radar Cross Section of targets located at range distances up to $150 \mathrm{~m}$;

(b) Vibration spectrum of targets 

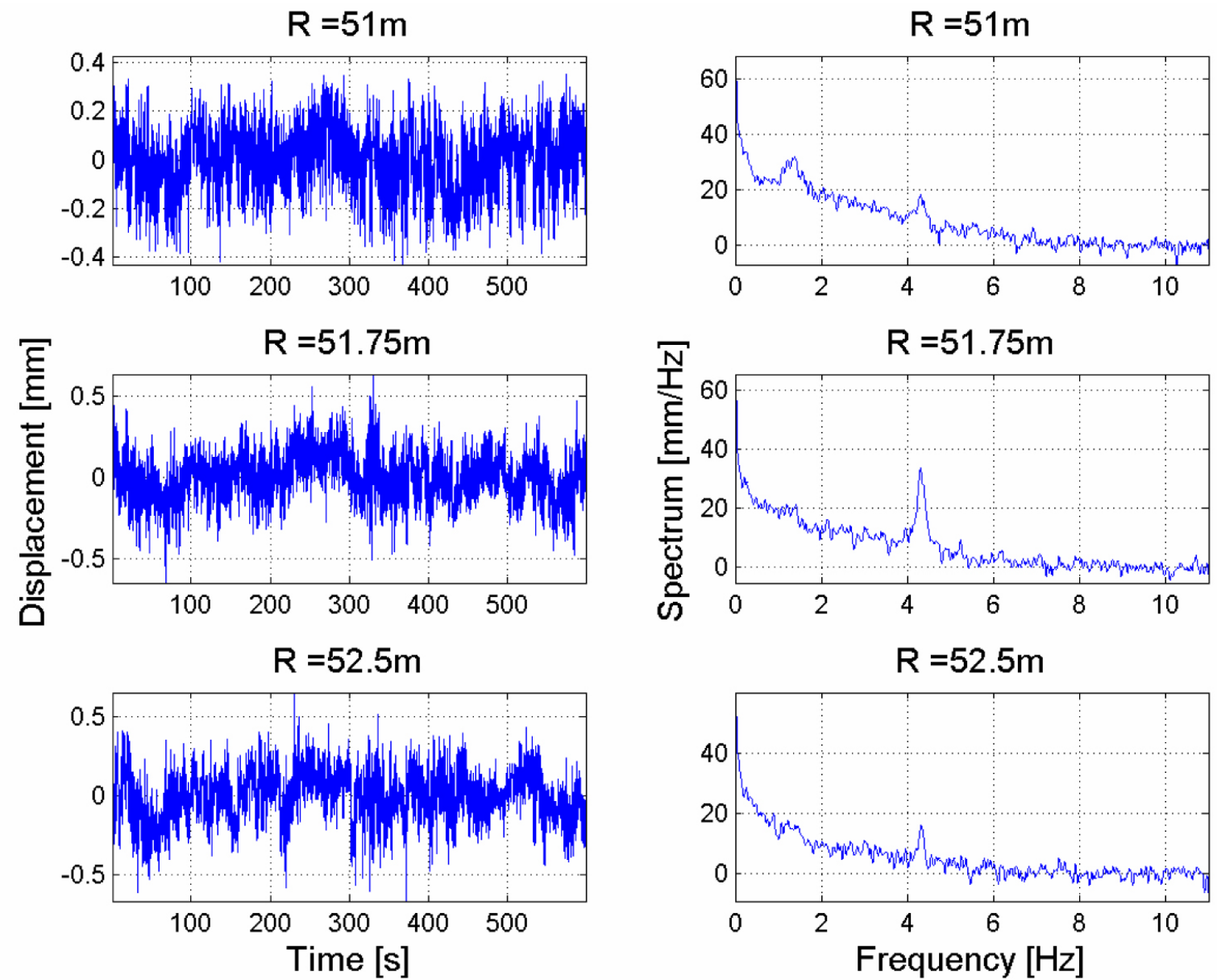

Fig. 8. Orvieto Cathedral façade, GBSAR installation site 2:

displacement profiles and the corresponding vibration spectra of selected targets

\section{REFERENCES}

1. Castagnetti C., Cosentini R.M., Lancellotta R., Capra A. Geodetic monitoring and geotechnical analyses of subsidence induced settlements of historic structures. Structural Control Health Monitoring. 2017. V. 24 (12). P. 1-15. DOI: 10.1002/stc. 2030.

2. Chen F., You J., Tang P. et al. Unique performance of spaceborne SAR remote sensing in cultural heritage applications: Overviews and perspectives. Archaeological Prospection. 2018. V. 25. P. 71-79. DOI: 10.1002/arp.1591.

3. Fanti R., Gigli G., Lombardi L. et al. Terrestrial laser scanning for rockfall stability analysis in the cultural heritage site of Pitigliano (Italy). Landslides. 2013. V. 10. P. 409-420. DOI: 10.1007/s.10346-012-0329-5.

4. Masini N., Persico R., Rizzo E. Some examples of GPR prospecting for monitoring of the monumental heritage. Journal of Geophysics and Engineering. 2010. V. 7. P. 190-199. DOI: $10.1088 / 1742-2132 / 7 / 2 / \mathrm{S} 05$.

5. Di Pasquale A., Nico G., Pitullo A., Prezioso G. Monitoring strategies of earth dams by ground-based radar interferometry: how to extract useful information for seismic risk assessment. Sensors. 2018. V. 18 (244). P. 244-271. DOI: 10.3390/s18010244.

6. Pieraccini M., Dei D., Betti M. et al. Dynamic identification of historic masonry towers through an expeditious and no-contact approach: Application to the "Torre del Mangia" in Siena (Italy). Journal of Cultural Heritage. 2015. V. 15. P. 275-282. DOI: 10.1016/j.culher.2013.07.006. 